EPJ Web of Conferences 72, 00019 (2014)

DOI: 10.1051/epjconf / 20147200019

(C) Owned by the authors, published by EDP Sciences, 2014

\title{
Physics with the Belle detector
}

\author{
Pavel Pakhlov ${ }^{1,2, a}$ \\ ${ }^{1}$ Institute for Theoretical and Experimental Physics, Moscow, Russia \\ ${ }^{2}$ Moscow Institute of Physics and Technology, Moscow region, Russia
}

Abstract. A summary of the experimental results obtained in the Belle experiment and future prospects of heavy flavor physics at the super B factory is presented.

\section{Introduction}

The Standard Model (SM) is one of the best experimentally verified physics theories. Since its establishment the SM managed to overcome all experimental tests and provide a good description for all processes in a wide energy range up to the scale probed in energy frontier experiments at LHC. The observation of the Higgs boson [1], the last fundamental particle of the SM that escaped the detection for decades, marked a triumph of the Standard Model.

Despite the great success in describing the matter and forces of nature, the SM is obviously not a complete theory. It fails to resolve some intrinsic problems, including the instability of the fundamental scale of weak interactions against radiative corrections, and leaves unanswered many fundamental questions, such as origin of gauge groups with independent coupling constants, fermion mass and mixing hierarchy etc. This suggests that the SM is only an effective theory valid up to a certain accuracy of tests or up to some energy scale. It is widely believed that the physics beyond the SM can be observed in precision or energy frontier experiments in the near future.

The quark sector of the SM is particularly rich in the SM puzzles and the largest contributor in terms of number of free parameters of the model. This sector potentially can reveal New Physics (NP) effects, and the measuring of the quark mixing parameters provides a major test of this sector of the SM, in particular of the CabibboKobayashi-Maskawa (CKM) description of flavor changing currents [2] and $C P$ violation.

Although CKM mixing does provide an only source for $C P$ violation - one of the Sakharov's conditions for the evolution of a matter-dominated universe [3], the magnitude of the matter-antimatter asymmetry cannot be explained solely by the CKM mechanism. This may indicate that some hidden mechanism exists at a higher energy scale. Flavor physics is a promising tool for NP searches through quantum loop effects. Rare decays, neutral meson-antimeson mixing and $C P$ violation are poten-

\footnotetext{
ae-mail: pakhlov@itep.ru
}

tially subject to NP virtual corrections even if the NP scale is above those accessible at today experiments.

The major information about the CKM matrix can be obtained in the study of $B$ mesons, thus providing the stringent test of the SM in the quark sector. This was the main motivation for construction of two B factory experiments, Belle and BaBar. In the previous decade both experiments have performed many precise and independent measurements of the CKM parameters. The underlying idea is to check the overall consistency of the CKM framework; any significant discrepancy between various measurements could be interpreted as potential NP effects. These experiments have confirmed the complex phase of the CKM matrix as the main source of $C P$ violation with high precision. Future experiments at the Super Flavor Factories, such as the Belle II experiment at SuperKEKB, are scheduled to address the open questions and perform even more precise tests.

\section{Belle experiment}

The Belle detector [4], located at the collision point of the $e^{+} e^{-}$asymmetric-energy collider KEKB, is a large-solidangle magnetic spectrometer. Belle was designed and optimized for the observation of $C P$ violation in the $B$ meson system. Its large coverage, precise vertex and momentum measurement for charged tracks, good particle identification, and few-percent precision electromagnetic calorimetry as well as effective muon and $K_{L}^{0}$ detection allow for many other searches apart from the study of $C P$ violation in $B$ decays. Plenty of outstanding results are attributable both to the good detector operation and to the excellent performance of the KEKB collider. A world-record luminosity of $2.1 \times 10^{34} \mathrm{~cm}^{-2} \mathrm{~s}^{-1}$, more than twice the design luminosity, was achieved for the KEKB accelerator. Before stopping the operation in 2010 to give way to the upgrade, the Belle experiment collected more than $1 \mathrm{ab}^{-1}$ of data over various bottomonium resonances. The Belle data sample is the world largest for the $\Upsilon(2 S), \Upsilon(4 S)$ and $\Upsilon(5 S)$ resonances. 
The results presented here are mostly based on the analysis of the full $\Upsilon(4 S)$ data sample, corresponding to $772 \times 10^{6} B \bar{B}$ pairs, collected with the Belle detector.

\section{Unitarity Triangle measurements at Belle}

The unitary relations of the CKM matrix can be represented as Unitarity Triangles (UT) in the complex plane. One of these triangles, that visualizes the unitarity relation between the first and third columns of the CKM matrix,

$$
V_{t d} V_{t b}^{*}+V_{c d} V_{c b}^{*}+V_{u d} V_{u b}^{*}=0
$$

is the most important for $C P$ violation studies in $B_{d}$ decays (see Fig. 1). Many of the important results described below can be depicted as constraints in this UT, including measurements of its angles and lengths of its sides.

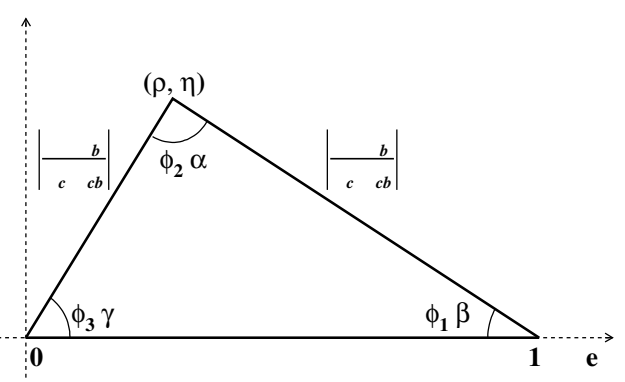

Figure 1. Unitarity triangle.

The measurement of two angles $\left(\phi_{1}\right.$ and $\left.\phi_{2}\right)$ is based on the extracting the amplitudes of the indirect (arising due to interference between decays with and without mixing) $C P$ violation asymmetry in particular decay modes. Such measurements require to determine the difference of lifetimes of two $B$ mesons, coherently produced in $\Upsilon(4 S)$ decays. In energy-asymmetric $e^{+} e^{-}$collisions at KEKB, the $\Upsilon(4 S)$ is produced with a Lorentz boost of $\beta \gamma=0.425$ along the beam axis. Since $B$ mesons are approximately at rest with respect to the $\Upsilon(4 S)$, we can measure $\Delta t$ by measuring the displacement between the two $B$ meson decay vertices in the $z$ direction.

\section{$3.1 \sin \left(2 \phi_{1}\right)$ from $B^{0} \rightarrow(c \bar{c}) K^{0}$}

The most precise determination of the angle $\phi_{1}$ (also known as $\beta$ ) is provided by the measurement of the mixing-induced $C P$ violation in $B^{0} \rightarrow(c \bar{c}) K^{0}$ decays:

$$
\begin{aligned}
A_{C P}(\Delta t) & =\frac{N\left(\bar{B}^{0} \rightarrow(c \bar{c}) K^{0}\right)-N\left(B^{0} \rightarrow(c \bar{c}) K^{0}\right)}{N\left(\bar{B}^{0} \rightarrow(c \bar{c}) K^{0}\right)+N\left(B^{0} \rightarrow(c \bar{c}) K^{0}\right)} \\
& =\sin 2 \phi_{1} \sin \left(\Delta m_{d} \Delta t\right)+A \cos \left(\Delta m_{d} \Delta t\right) .
\end{aligned}
$$

These decays, often referred to as "golden modes", are dominated by the Cabibbo-favored tree diagram $b \rightarrow c \bar{c} s$ with an internal $W$ boson emission. The leading penguin contribution to these final states has the same weak phase within a few per cent accuracy. This makes the second term in Eq. 2 vanishing to a very good approximation. Besides theoretical clarity, these channels also offer experimental advantages because of the relatively large branching fractions $\left(\sim 10^{-3}\right)$ and the presence of narrow resonances in the final state, which provides a powerful suppression of combinatorial background.

Belle has presented the final result on $\sin \left(2 \phi_{1}\right)$ using the full $\Upsilon(4 S)$ data set [5]. In addition to more data, significant enhancement in the reconstruction efficiency with the improved track reconstruction algorithm allows one to get the record statistical accuracy of the result. Moreover, special efforts were made to reduce the systematics errors of the measurement. The $C P$ eigenstates considered in the analysis are $J / \psi K_{S}^{0}, \psi(2 S) K_{S}^{0}, \chi_{c 1} K_{S}^{0}(C P \approx$ odd $)$, and

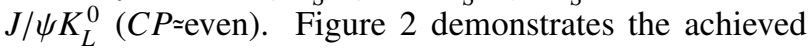
signal purity. For the selected candidate events the neutral $B$ flavor $(q= \pm 1)$ is identified from the decay products of the accompanying $B$ meson based on the information on the charge of daughter leptons, kaons, baryons, and pions.
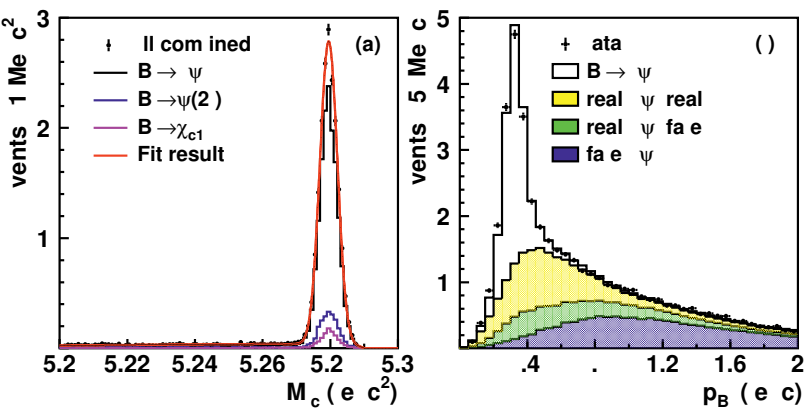

Figure 2. a) $M_{b c}$ distribution for $B^{0} \rightarrow J / \psi K_{S}^{0}$ (black), $\psi(2 S) K_{S}^{0}$ (blue), and $\chi_{c 1} K_{S}^{0}$ (magenta); the superimposed red curve shows the fit result for all modes combined. b) The $p_{B}^{*}$ distribution for $B^{0} \rightarrow J / \psi K_{L}^{0}$ candidates with the results of the fit (open histogram), and different background sources.

Figure 3 shows the $\Delta t$ distributions and timedependent asymmetries for good flavor tag quality events for both $C P$ odd and $C P$ even final states. The sign of the asymmetry for the opposite $C P$ eigenvalues is flipped, as expected. The observed asymmetry picture is consistent with the absence of the direct $C P$ violation.

The measured $C P$ violation parameters from the Eq. 2 are $\sin \left(2 \phi_{1}\right)=0.667 \pm 0.023$ (stat) \pm 0.012 (syst) and $A=$ $0.006 \pm 0.016$ (stat) \pm 0.012 (syst). This represents the most precise determination of mixing-induced $C P$ violation in a $B$ meson decay, and hence provides a solid reference point for the SM that can be used to search for evidence of NP.

\subsection{Measurement of the angle $\phi_{2}$}

This angle can be determined by measuring a timedependent $C P$ asymmetry in charmless $b \rightarrow u \bar{u} d$ decays such as $B^{0} \rightarrow \pi^{+} \pi^{-}$. The weak decay phase of $b \rightarrow u$ transition (Fig. 4, left) is related to $V_{u b}$ element, thus the time dependent $C P$ asymmetry in pure $b \rightarrow u$ modes is equal to $\sin 2 \phi_{2}$. However, an additional penguin amplitude (Fig. 4, right) contributes with a phase that is different from the 

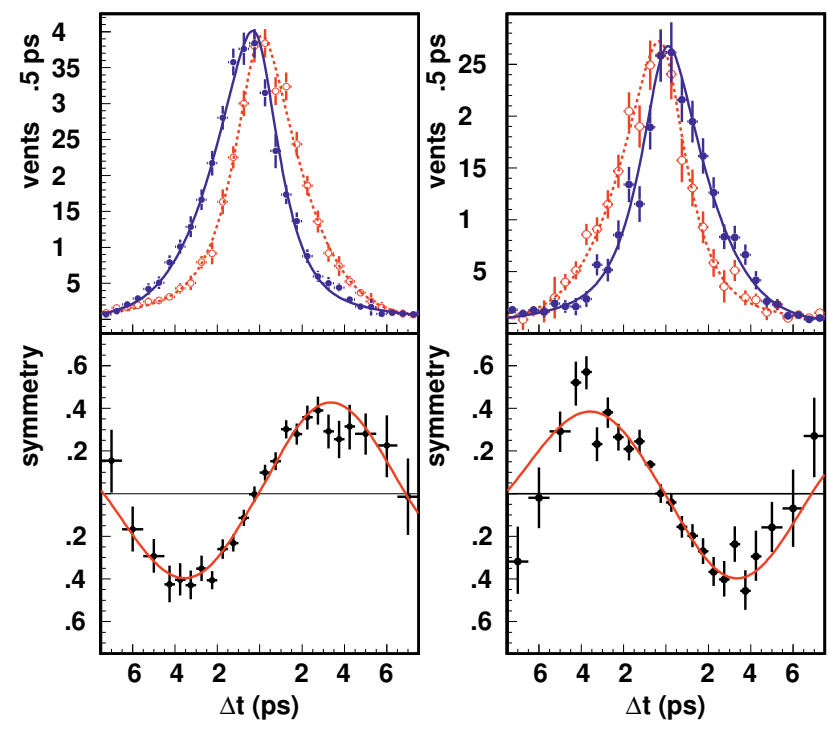

Figure 3. The background-subtracted $\Delta t$ distribution (top) for $q=+1$ (red) and $q=-1$ (blue) $B^{0} \rightarrow(c \bar{c}) K^{0}$ events and asymmetry (bottom) for good tag quality events for all $C P$-odd modes combined (left) and the $C P$-even mode (right).

tree diagram $\left(V_{t d}\right.$ instead of $\left.V_{u b}\right)$. This causes a deviation of the amplitude of indirect $C P$ asymmetry from $\sin 2 \phi_{2}$ and a non-zero direct $C P$ asymmetry. The angle $\phi_{2}$ can be extracted following the idea of M. Gronau and D. London [6] using the isospin relation among branching fractions and $C P$ asymmetries of $B^{0} \rightarrow \pi^{+} \pi^{-}, B^{0} \rightarrow \pi^{0} \pi^{0}$, and $B^{+} \rightarrow \pi^{+} \pi^{0}$ decays. The method allows to constrain the contribution from the penguin amplitude generally with an eight-fold ambiguity.

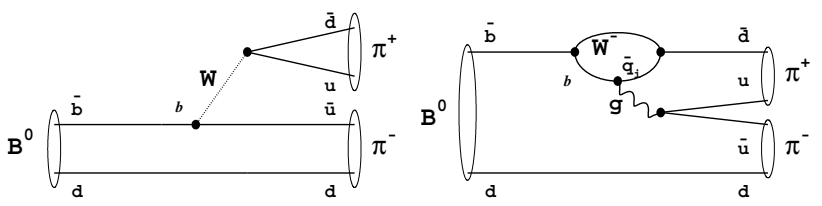

Figure 4. Feynman diagrams for $B^{0} \rightarrow \pi^{+} \pi^{-}$decays.

This decay $B^{0} \rightarrow \pi^{+} \pi^{-}$has the simplest two-body topology and was the first with well established $C P$ asymmetry [7]. However, the large observed direct $C P$ violation, and large branching fraction for $B^{0} \rightarrow \pi^{+} \pi^{-}$[8] suggest that the penguin contribution to this final state is large, thus complicating the extraction of $\phi_{2}$. Using the full data set Belle measured the $C P$ asymmetry in $B^{0} \rightarrow \pi^{+} \pi^{-}$quite precisely [9]: $A=+0.33 \pm 0.06 \pm 0.03$ and $S=-0.64 \pm 0.08 \pm 0.03$ (the world's most precise measurement of time-dependent $C P$ violation parameters in $B^{0} \rightarrow \pi^{+} \pi^{-}$!). Figure 5 shows the observed $\Delta t$ distributions for the $B^{0} \rightarrow \pi^{+} \pi^{-}$events tagged as $B^{0}$ and $\bar{B}^{0}$, and observed asymmetry. However, because of trigonometrical ambiguities in extraction of $\phi_{2}$ (see Fig. 6), Belle only managed to exclude the region $23.8^{\circ}<\phi_{2}<66.8^{\circ}$ at $68 \%$ CL from this modes.

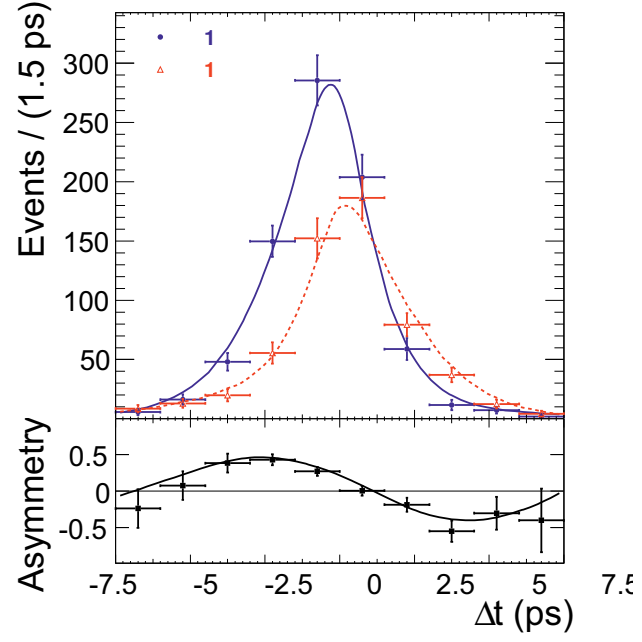

Figure 5. Background subtracted time-dependent fit results for $B^{0} \rightarrow \pi^{+} \pi^{-}$. Top: the $\Delta t$ distribution for $\bar{B}^{0}$ (red) and $B^{0}$ (blue) tags. Bottom: asymmetry for good tag quality events.

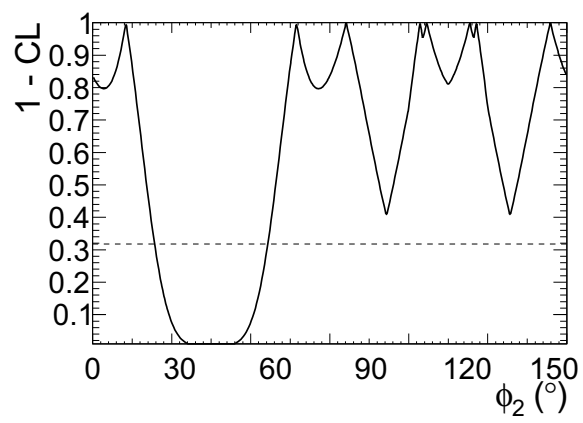

Figure 6. Difference $1-C L$ for a range of $\phi_{2}$ from Belle's $B \rightarrow$ $\pi \pi$ analysis.

We are lucky to get quite high sensitivity on $\phi_{2}$ from the $B \rightarrow \rho \rho$ decays, where the penguin contribution turns out to be small. This final state is much more difficult for a experimental study because of two wide vector mesons in the final state. As the $C P$ eigenvalue for $B^{0} \rightarrow \rho^{+} \rho^{-}$ is $(-1)^{L}(L=0,1,2)$, it is necessary to separate the two opposite $C P$ components through an angular analysis. The later demonstrates that a $C P$-even component dominates, and the opposite component can be safely ignored. The constraint on this angle from the last Belle $B \rightarrow \rho \rho$ study [10] with the full data set is $\phi_{2}=(84.9 \pm 12.9)^{\circ}$.

Determination of $\phi_{2}$ is also possible using the decays $B^{0} \rightarrow \rho^{ \pm} \pi^{\mp}$, in spite of the final states are not $C P$ eigenstates. To measure $\phi_{2}$ from $B \rightarrow \rho \pi$ it is required to measure four isospin amplitudes leading to 12 unknowns in the isospin pentagon. The problem is simplified and (what is even more important) the ambiguity introduced by geometry of isospin triangles is removed with the time-dependent Dalitz plot analysis of the $\pi^{+} \pi^{-} \pi^{0}$ final state, which includes all the $B^{0} \rightarrow(\rho \pi)^{0}$ decays [13]. Belle performed such a study using the statistics of $449 \times 10^{6} B \bar{B}$ events and 
derived the constraint $68^{\circ}<\phi_{2}<95^{\circ}$ at $68 \% \mathrm{CL}$ for the solution consistent with the SM [11].

The current world average of $\phi_{2}$ [12] including measurements of these three modes from Belle and BaBar, $\left(85.4_{-3.8}^{+4.0}\right)^{\circ}$, is quite precise. However, for $B \rightarrow \rho \pi$ modes Belle has not still published the final result with the full data set, and it is important to have these updated results soon.

\subsection{Measurement of the angle $\phi_{3}$}

The UT angle $\phi_{3}$ relies on the measurement of direct $C P$ violation in $B^{+} \rightarrow D^{0} K^{+}$decays caused by interference between the two contributing amplitudes with different CKM phases (Fig. 7), if both $D^{0}$ and $\bar{D}^{0}$ mesons decay to a common final state. The method is theoretically clean due to the absence of loop contributions, however, the color suppressed amplitude (Fig. 7, right) is almost an order of magnitude smaller, hence resulting in a small $C P$ asymmetry, which complicates the extracting of $\phi_{3}$. There are basically three methods to measure: The GLW method [14] uses the $D^{0}$ decays in $C P$-even and $C P$-odd final states like $K^{+} K^{-}$or $K_{S}^{0} \pi^{0}$. The ADS method [15] is based on doubly-Cabibbo suppressed decays like $D^{0} \rightarrow K^{+} \pi^{-}$; The GGSZ method [16] is based on a Dalitz plot analysis of the three-body decays of $D^{0}$ decays such as $D^{0} \rightarrow K_{S}^{0} \pi^{+} \pi^{-}$. The last method provides the highest statistical power for measuring $\phi_{3}$.

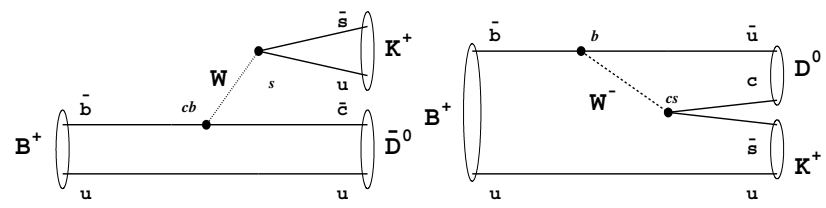

Figure 7. Feynman diagrams for $B^{+} \rightarrow D^{0} K^{+}$decays.

In the GGSZ method the amplitude for $B^{+} \rightarrow$ $D^{0}\left(K_{S}^{0} \pi^{+} \pi^{-}\right) K^{+}$decay as a function of Dalitz plot variables $m_{+}^{2}=m_{K_{S}^{0} \pi^{+}}^{2}$ and $m_{-}^{2}=m_{K_{S}^{0} \pi^{-}}^{2}$ is given by

$$
f_{B}^{+}=f_{D}\left(m_{+}^{2}, m_{-}^{2}\right)+r_{B} e^{i \phi_{3}+i \delta} f_{D}\left(m_{-}^{2}, m_{+}^{2}\right),
$$

where $f_{D}\left(m_{+}^{2}, m_{-}^{2}\right)$ is the amplitude of the $\bar{D}^{0} \rightarrow K_{S}^{0} \pi^{+} \pi^{-}$ decay, $r_{B}$ is a ratio of two amplitudes, and $\delta$ is a strong phase difference. Similarly, the amplitude for $B^{-} \rightarrow$ $D^{0}\left(K_{S}^{0} \pi^{+} \pi^{-}\right) K^{-}$decay is

$$
f_{B}^{-}=f_{D}\left(m_{-}^{2}, m_{+}^{2}\right)+r_{B} e^{-i \phi_{3}+i \delta} f_{D}\left(m_{+}^{2}, m_{-}^{2}\right) .
$$

Once $f_{D}$ is fixed, a simultaneous fit to $B^{+}$and $B^{-}$data allows to extract $\phi_{3}, r_{B}$ and $\delta$ separately.

The $D^{0} \rightarrow K_{S}^{0} \pi^{+} \pi^{-}$decay amplitude can be determined from a large sample of flavor-tagged $D^{*+} \rightarrow D^{0} \pi^{+}$ decays produced in continuum $e^{+} e^{-}$annihilation. However, with this approach the description of $f_{D}$ is based on a model, that includes interfering resonances in $K_{S}^{0} \pi^{+}$, $K_{S}^{0} \pi^{-}$and $\pi^{+} \pi^{-}$systems as well as a nonresonant term.
Using the GGSZ method with a model-dependent description of $f_{D}$, Belle's earlier measurement [17] based on a data sample of $605 \mathrm{fb}^{-1}$ integrated luminosity yielded $\phi_{3}=$ $\left(78.4_{-11.6}^{+10.8} \pm 3.6 \pm 8.9\right)^{\circ}$ and $r_{B}=0.160_{-0.038}^{+0.040} \pm 0.011_{-0.010}^{+0.050}$, where the last error comes from the Dalitz plot model uncertainty.

While the statistical errors of the GGSZ method will be reduced with an increased data sample, the accuracy will be still limited by the model uncertainty. The new approach was tested by Belle [18] to fight this seeming irreducible bound of the method. Instead of using a parametrized $f_{D}$ function, Belle substituted the Dalitz plot distribution taken directly from the data obtained by CLEO [19] from the decays of quantum-correlated $D^{0} \bar{D}^{0}$ pairs produced in the $\psi(3770)$. In contrast to the previous method, the model-independent approach has to utilize a binned Dalitz plot. From the a combined fit Belle obtained $\phi_{3}=\left(77.3_{-14.9}^{+15.1} \pm 4.1 \pm 4.3\right)^{\circ}$ and $r_{B}=0.145 \pm$ $0.030 \pm 0.010 \pm 0.011$, where the last error is due to limited precision of CLEO data. The significance of direct $C P$ violation is 2.7 standard deviations. Compared to results of the model-dependent method, the last measurement has slightly poorer statistical precision mostly because of a smaller $r_{B}$ in this analysis. However, the large model uncertainty for the model-dependent study $\left(8.9^{\circ}\right)$ is replaced by a purely statistical uncertainty due to the limited size of the CLEO data sample $\left(4.3^{\circ}\right)$, which in future can be reduced with the BESIII or Super Charm-Tau factory data. The model-independent approach therefore offers a perspective course for studies at Belle II.

Two other methods (GLW and ADS) were also used in Belle analysis [20] and are useful for tighter constraints on both $\phi_{3}$ and the hadronic parameters for a more reliable measurement. Belle results on $\phi_{3}$ are in agreement with those obtained by BaBar and LHCb. The world average results on $\phi_{3}$ is $\left(68.0_{-8.5}^{+8.0}\right)^{\circ}$ [12].

\subsection{UT summary}

Belle performed plenty of analyses to constrain the sides of the UT as well. These topics due to hugeness of the related material is outside of the scope of this review. We summarize the UT studies by illustrating the constraints obtained mostly by two B factory experiments, Belle and BaBar, with Fig. 8 produced by the CKMfitter collabora-

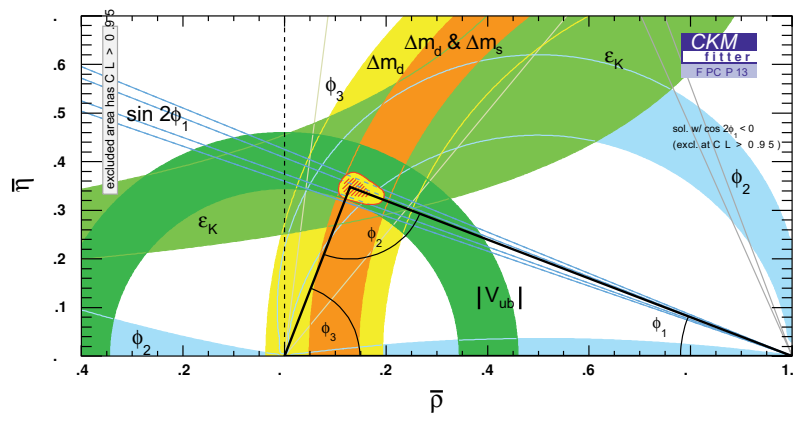

Figure 8. Constraints on the UT as compiled by CKMfitter. 
tion [12]. Each colored band here corresponds to a different kind of process, and looking closely we can see quite nice agreement between independent constraints. It is worth mentioning that thanks to BaBar and Belle the square of allowed area for the position of the UT upper apex is squeezed by two orders of magnitudes compared to the pre- $\mathrm{B}$ factory era.

\section{$4 C P$ violation in penguin dominated modes}

It is widely believed that $B$ meson penguin decays can serve as one of the most sensitive probes for NP due to a possible non-SM contribution (e.g. from SUSY particles) in the loop diagram. In particular, manifestations of the NP contribution in the penguin dominated modes can be revealed as deviations of $C P$ violation parameters from the SM expectations. In $b \rightarrow s \bar{q} q$ hadronic decays, the $\mathrm{SM}$ weak phase is the same as in the $B^{0} \rightarrow(c \bar{c}) K^{0}$ transition. Therefore, the main task is to check whether the penguin $C P$ violation parameter $\sin 2 \phi_{1}^{\text {eff }}$ is equal to $\sin 2 \phi_{1}$, and the direct $C P$ violation is absent $(A=0)$. However, there are some SM corrections to these relations coming from the Cabibbo-suppressed tree diagram, final state interaction effects, etc. Theoretical calculations predict the uncertainties are of the order of $1 \%$ for $b \rightarrow s \bar{s} s$, and up to $10 \%$ for other $b \rightarrow s \bar{q} q$ modes.

Earlier Belle measurements of $C P$ asymmetries in $B^{0} \rightarrow \phi K_{S}^{0}$, using a $152 \times 10^{6} B \bar{B}$ data sample [21], showed an exciting $3.5 \sigma$ deviation of $\sin 2 \phi_{1}^{\text {eff }}$ from $\sin 2 \phi_{1}$. The measurements updated in three years with almost three times higher statistics [22] showed already quite good (but disappointing) agreement. The results of several recent Belle studies are summarized in Table 1 . These are consistent with the $C P$ violation in $B^{0} \rightarrow(c \bar{c}) K^{0}$ decays at the $1 \sigma$ level.

Table 1. Measurements of $C P$ violation parameters, $\sin 2 \phi_{1}^{\mathrm{eff}}$ and $A$, in $B^{0} \rightarrow \eta^{\prime} K^{0}, \phi K^{0}$, and $K_{S}^{0} K_{S}^{0} K_{S}^{0}$ modes with a $535 \times 10^{6} B \bar{B}$ data sample.

\begin{tabular}{lcc}
\hline \hline mode & $\sin 2 \phi_{1}^{\text {eff }}$ & $A$ \\
\hline$\eta^{\prime} K^{0}$ & $+0.64 \pm 0.10 \pm 0.04$ & $-0.01 \pm 0.07 \pm 0.05$ \\
$\phi K^{0}$ & $+0.50 \pm 0.21 \pm 0.06$ & $+0.07 \pm 0.15 \pm 0.05$ \\
$K_{S}^{0} K_{S}^{0} K_{S}^{0}$ & $+0.30 \pm 0.32 \pm 0.08$ & $-0.31 \pm 0.20 \pm 0.07$ \\
\hline \hline
\end{tabular}

Including other $b \rightarrow s$ mediated $B$ decays, the precision of $\sin 2 \phi_{1}^{\text {eff }}$ is still statistically limited, typically $0.1-0.2$. Obtaining a $1 \%$ level sensitivity which provides already a real probe for the NP requires a Super B factory experiment.

\section{Rare $B$ decays}

Prefacing this chapter it is worth to remind that precise measurements of rare decays, i.e. processes suppressed in the SM, are sensitive to NP at scales that can exceed those that achievable at the energy frontier. This is demonstrated by a few analyses described below involving both loop and tree decay diagrams.

\section{$5.1 \quad b \rightarrow s \gamma$}

The dominant contribution in the SM to $b \rightarrow s \gamma$ decays is from a loop involving the top quark and $W$ boson. The measurement of the inclusive $b \rightarrow s \gamma$ branching fraction gives access to the value of $V_{t s}$, but, what is more important, it provides a tool to search for and constrain physics beyond the SM. Indeed, the SM particles in the loop may be replaced by hypothetical particles such as the charged Higgs boson or SUSY particles resulting in observable deviations of the decay rate.

The inclusive $b \rightarrow s \gamma$ rate (i.e. summed over all possible strange hadronic states) suffers from the minimal theoretical uncertainty in contrast to the rates of particular exclusive radiative channels, where the hadronic uncertainties are large. $\mathcal{B}\left(B \rightarrow X_{s} \gamma\right)$ is calculated within the SM including next-to-next-to-leading logarithmic corrections with a $\sim 7 \%$ precision. Ideally, the inclusive photon spectrum should be measured over the entire energy range. Practically, the lower part of the spectrum is hardly accessible due to insurmountably large background. Even in the intermediate energy region the continuum background is several orders of magnitude larger than the inclusive photon signal. The continuum background is subtracted using the Belle's off-resonance data sample. The obtained spectrum is shown in Fig. 9 and the branching fraction was measured to be

$$
\mathcal{B}\left(B \rightarrow X_{s} \gamma ; E_{\gamma}>1.7 \mathrm{GeV}\right)=(3.45 \pm 0.15 \pm 0.40) \times 10^{-4}
$$

The world average [25] (using the analogous BaBar measurement) extrapolated for $E_{\gamma}>1.6 \mathrm{GeV}$ is $\mathcal{B}\left(B \rightarrow X_{s} \gamma\right)=$ $(3.55 \pm 0.24(\exp ) \pm 0.09($ model $)) \times 10^{-4}$. This is consistent with the theoretical prediction, and has been used to constrain NP scenarios. For example, the charged Higgs mass is bounded from this measurement to be above $295 \mathrm{GeV}$.

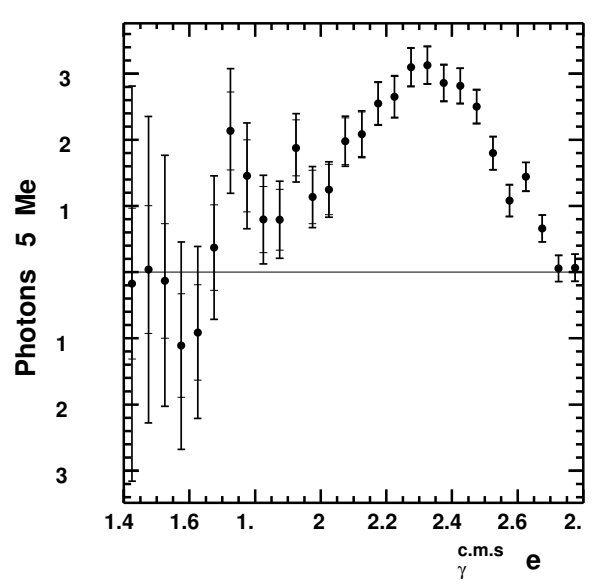

Figure 9. Photon energy spectrum from $B \rightarrow X_{s} \gamma$.

\subsection{Measurement of the $\mathcal{B}\left(B^{+} \rightarrow \tau^{+} v\right)$}

The leptonic $B^{+} \rightarrow \tau^{+} v$ decay and the semileptonic $B \rightarrow$ $D^{(*)} \tau^{+} \bar{v}$ decays are sensitive to the NP including the SM 
extensions with charged Higgs bosons, that could significantly suppress or enhance the branching ratios for these decays. The charged Higgs mass is not strongly constrained by the branching ratio of the $b \rightarrow s \gamma$ process due to possible destructive interference between diagrams involving lopps with SUSY particles and the charged Higgs boson. Experimentally it is a real challenge to identify modes with a $\tau$ lepton due to multiple neutrinos in the final state. At the $e^{+} e^{-}$B factories, exclusive production of a $B$ meson pair with no extra particles allows to tag the signal decay by reconstruction of all particles originating from the accompanying $B$ meson. Using the hermeticity of the detector it is possible to identify the signal as absence of the energy deposited in the detector not associated with the tag and signal particles. Two sorts of decays are employed for reconstructing of the tagging $B$ : hadronic decays such as $B \rightarrow D \pi$ ("hadronic tag") and semileptonic decays such as $B \rightarrow D \ell v$ ("semileptonic tag"). Both methods provide similar sensitivity: while the efficiency is higher for the semileptonic tag, the hadronic tag provides better purity.

The first evidence for $B^{+} \rightarrow \tau^{+} v$ was reported by Belle using a hadronic tag and a data sample corresponding to $449 \times 10^{6} B \bar{B}$ events [26]. This was followed by a measurement using a semileptonic tag and a data sample corresponding to $657 \times 10^{6} B \bar{B}$ [27]. The branching fraction obtained by the semileptonic-tag analysis is $\mathcal{B}\left(B^{+} \rightarrow \tau^{+} v\right)=\left(1.54_{-0.37-0.31}^{+0.38}\right) \times 10^{-4}$ with the significance of $3.6 \sigma$. The hadronic-tag result has been updated using the Belle's final sample [28]. The branching fraction is found to be $\mathcal{B}\left(B^{+} \rightarrow \tau^{+} v\right)=\left(0.72_{-0.25}^{+0.27} \pm 0.11\right) \times 10^{-4}$ with a significance of $3.0 \sigma$ (Fig. 10). In the last paper, by employing a neural network-based method for the hadronic tag and a two-dimensional fit for the signal extraction, along with a larger data sample, both statistical and systematic precision are significantly improved. Combining the semileptonic-tag and hadronic-tag results taking into account all the correlated systematic uncertainties, the branching fraction is found to be $(0.96 \pm 0.26) \times 10^{-4}$ with a significance of $4.0 \sigma$ [28].

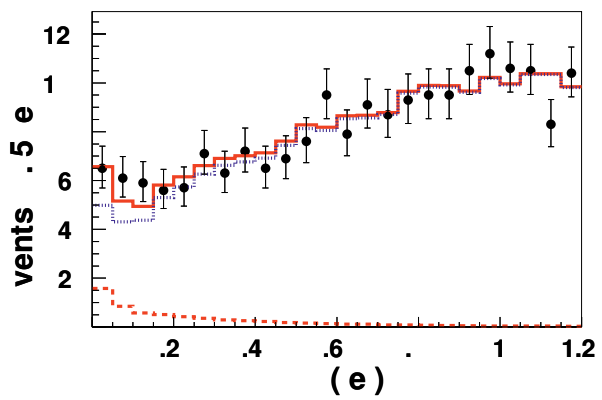

Figure 10. The residual unassociated energy deposited in the electromagnetic calorimeter. The red solid histograms show the projections of the fits. The red dashed and blue dotted histograms show the signal and background components, respectively.

The Belle result is in agreement with the BaBar measurement [29]. The world average value of $\mathcal{B}\left(B^{+} \rightarrow\right.$ $\left.\tau^{+} v\right)=(1.15 \pm 0.23) \times 10^{-4}$ is consistent (the deviation is $1.6 \sigma)$ with the $\mathrm{SM}$ expectation of $\mathcal{B}\left(B^{+} \rightarrow \tau^{+} v\right)=$ $\left(0.73_{-0.07}^{+0.12}\right) \times 10^{-4}$ obtained from other experimental constraints [12]. The measured $\mathcal{B}\left(B^{+} \rightarrow \tau^{+} v\right)$ sets constraints on the parameters of various models involving charged Higgs bosons. For large $\tan \beta$ the excluded region are stringent than those obtained from the direct searched at LHC.

\subsection{Measurement of the $\mathcal{B}\left(B \rightarrow D^{(*)} \tau^{+} v\right)$}

The SM predicts a $B \rightarrow D^{(*)} \tau^{+} v$ branching fraction of $1.4 \%$, i.e. strictly speaking these modes are not rare. The result is presented in terms of $R\left(D^{(*)}\right)=\mathcal{B}(B \rightarrow$ $\left.D^{(*)} \tau^{+} v\right) / \mathcal{B}\left(B \rightarrow D^{(*)} \ell v\right)$, which is independent of the $V_{c b}$ and of the parameterization of the strong interaction and can be compared with the SM theoretical expectations.

In two succeeding $B \rightarrow D^{(*)} \tau^{+} v$ analyses Belle used "inclusive" hadronic tag with much looser selection compared to the $B^{+} \rightarrow \tau^{+} v$ study with hadronic tag. The $B \rightarrow D^{(*)} \tau^{+} v$ decay was first observed by Belle for neutral $B$ mesons using the $535 \times 10^{6} B \bar{B}$ data sample [30]. The results for charged $B$ meson decays was obtained three years later using the $657 \times 10^{6} B \bar{B}$ data sample [31]. The decay $B^{+} \rightarrow \bar{D}^{* 0} \tau^{+} v_{\tau}$ is observed with a significance of 8.1 standard deviations, while only $3.5 \sigma$ significance was achieved for the decay $B^{+} \rightarrow \bar{D}^{0} \tau^{+} v_{\tau}$. Finally, Belle also obtained a preliminary result for all four modes using the $657 \times 10^{6} B \bar{B}$ data sample with exclusive hadronic tag [32].

The naive averages of $R\left(D^{(*)}\right)$ for the results mentioned above are $R(D)=0.430 \pm 0.091$ and $R\left(D^{*}\right)=$ $0.40 \pm 0.047$. For these estimates, the correlations in the statistical errors between the different tagging analyses are neglected as the overlap of events with two tagging methods is small. The results for $R\left(D^{(*)}\right)$ are consistent between the Belle and BaBar experiments [33]. The Belle results exceed the $\mathrm{SM}$ predictions $R(D)=0.297 \pm 0.017$ and $R\left(D^{*}\right)=0.252 \pm 0.003$ by $1.4 \sigma$ and $3.0 \sigma$, respectively. The BaBar results exceed these SM predictions by $2.0 \sigma$ and $2.7 \sigma$, respectively [33]. The combined disagreement between the experiment and the SM is at a $\gtrsim 4 \sigma$ level. It is worth mention that both results disfavor the type II twoHiggs doublet model at a level of more than $3 \sigma$ for the whole $\tan \beta$ region.

With larger statistics of Super B factory besides the improved accuracy of $R\left(D^{(*)}\right)$, the $q^{2}$ distributions and the angular distributions of the $\tau$ and $D^{(*)}$ decays could also provide useful information for testing the SM and constraining NP models.

\subsection{Rare decay summary}

Besides $B \rightarrow D^{(*)} \tau^{+} v$ modes, which show an intriguing excess over the SM predictions, other modes are in agreement with the expectations. These studies with present $\mathrm{B}$ factories statistics can provide the SM test a 10\% accuracy level. However, as we can see from the selectively described above analyses these measurement already probe the NP at an energy scale of $O(100 \mathrm{GeV})$. 


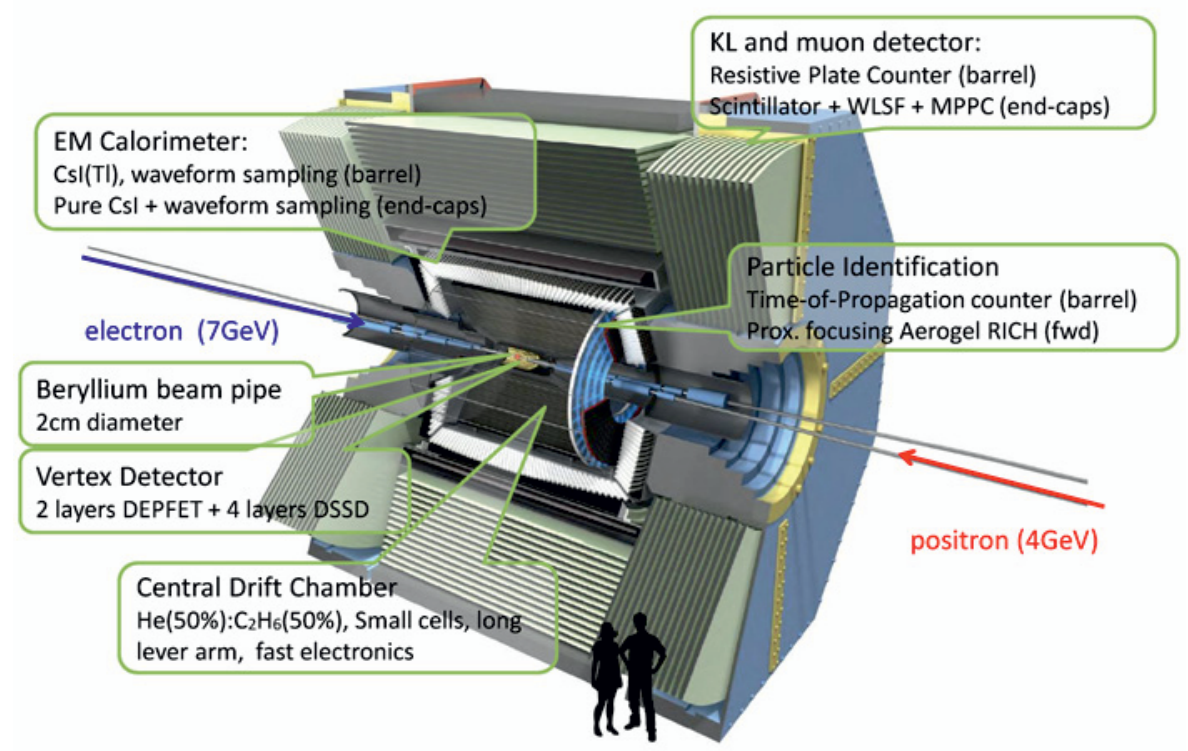

Figure 11. The Belle II detector layout.

\section{B physics Future}

The B factories have demonstrated that flavor physics has powerful potential to search for various NP manifestations. If statistical errors of measurements would be substantially improved, we could push the explored NP scale above the $1 \mathrm{TeV}$ region: up to now no obstacles to that were seen, such as irreducible systematics or theoretical uncertainty, that can preclude in moving forward with significantly increased data. Thereby, there is a strong motivation for construction of the Super B factory.

The idea of the Belle experiment upgrade was first presented in a Letter of Intent in 2004 [35], followed by a supplemental report in 2008 [36]. In parallel, the KEKB accelerator group has proposed the SuperKEKB machine, an upgrade of KEKB to increase the luminosity by two orders of magnitude, with an ultimate goal of $8 \times 10^{35} \mathrm{~cm}^{-2} \mathrm{~s}^{-1}$. Further work on the detector design resulted in the Technical Design report published in 2010 [34]. In March 2011 the accelerator as well as the detector upgrade were approved by the Japanese parliament and the project was launched.

Because of the increased level of background, the detector for new experiment (Belle II) has to cope with higher occupancy and radiation damage than the Belle detector. To be able to operate at the conditions of the SuperKEKB collider, the components of the Belle detector are either upgraded or replaced by new ones. Figure 11 shows the components of the Belle II detector and their main features. A detailed description of the detector can be found in Ref. [34].

The Belle II experiment has at least one important mission: to search for NP in the flavor sector exploiting a huge jump in luminosity and the plenty of independent measurements. If the NP will be observed at LHC before the start of Belle II, the flavor sector of NP still needs to be constrained, which is only possible at the Super B factory as many of such measurements can not be made at hadronic machines. The aim of the Belle II project is to accumulate $50 \mathrm{ab}^{-1}$, corresponding to about 55 billion $B \bar{B}$ pairs by the year 2022. The projected sensitivities for $50 \mathrm{ab}^{-1}$ are below 0.01 for $\sin 2 \phi_{1}$, less than $1^{\circ}$ for $\phi_{2}$, and $1.5^{\circ}$ for $\phi_{3}$. The accuracy in $C P$ violation studies, branching fractions and kinematics characteristics in rare $B$ decays will be also improved by an order of magnitude. The examples described above are only a small part of possible measurements to be performed with the Belle II experiment. A more detailed overview can be found in Ref. [37].

\section{References}

[1] G. Aad et al. (ATLAS Collaboration), Phys. Lett. B716, 1 (2012);

S. Chatrchyan et al. (CMS Collaboration), Phys. Lett. B716, 30 (2012).

[2] N. Cabibbo, Phys. Rev. Lett. 10, 531 (1963) 531; M. Kobayashi and T. Maskawa, Prog. Theor. Phys. 49, 652 (1973).

[3] A. D. Sakharov, Pisma Zh. Eksp. Teor. Fiz. 5, 32 (1967).

[4] S. Kurokawa et al., Nucl.Instrum.Meth. A499, 1 (2003);

A. Abashian et al., Nucl.Instrum.Meth. A479, 117 (2002).

[5] I. Adachi et al. (Belle Collaboration), Phys. Rev. Lett. 108, 171802 (2012).

[6] M. Gronau and D. London, Phys. Rev. Lett. 65, 3381 (1990).

[7] K. Abe, et al. (Belle Collaboration), Phys. Rev. D68, 012001 (2003).

[8] K. Abe, et al. (Belle Collaboration), Phys. Rev. Lett. 94, 181803(2005). 
[9] J. Dalseno et al. (Belle Collaboration), Phys. Rev. D88, 092003 (2013).

[10] P. Vanhoefer et al. (Belle Collaboration), arXiv:1212.4015.

[11] A. Kusaka et al. (Belle Collaboration), Phys. Rev. D77, 072001 (2008).

[12] J. Charles et al. (CKMfitter Group), Eur. Phys. J. C41, 1 (2005) and on-line update at http://ckmfitter.in2p3.fr/

[13] A.E. Synder and H.R. Quinn, Phys. Rev. D48, 2139 (1993).

[14] M. Gronau and D. London, Phys. Lett. B253, 483 (1991);

M. Gronau and D. Wyler, Phys. Lett. B265, 172 (1991).

[15] D. Atwood, I. Dunietz, A. Soni, Phys. Rev. Lett. 78, 3257 (1997);

Phys. Rev. D63, 036005 (2001).

[16] A. Giri, Y. Grossman, A. Soffer, J. Zupan, Phys. Rev. D68, 054018 (2003);

A. Bondar, Proceedings of BINP Special Analysis Meeting on Dalitz Analysis, 24-26 Sep. 2002, unpublished.

[17] A. Poluektov et al. (Belle Collaboration), Phys. Rev. D81, 112002 (2010).

[18] H. Aihara et al. (Belle Collaboration), Phys. Rev. D85, 112014 (2012).

[19] R. A. Briere et al. (CLEO Collaboration), Phys. Rev. D80, 032002 (2009).

[20] Y. Horii et al. (Belle Collaboration), Phys. Rev. Lett. 106, 231803 (2011).

[21] K. Abe et al. (Belle Collaboration), Phys. Rev. Lett. 91, 261602 (2003).

[22] K.-F. Chen et al. (Belle Collaboration), Phys. Rev. Lett. 98, 031802 (2007).
[23] Y. Nakahama et al. (Belle Collaboration), Phys. Rev. D82, 073011 (2010).

[24] A. Limosani et al. (Belle Collaboration), Phys. Rev. Lett. 103, 241801 (2009).

[25] Y. Amhis et al. (Heavy Flavor Averaging Group), arXiv:1207.1158 and online update at http://www.slac.stanford.edu/xorg/hfag.

[26] K. Ikado et al. (Belle Collaboration), Phys. Rev. Lett. 97, 251802 (2006).

[27] K. Hara et al. (Belle Collaboration), Phys. Rev. D82, 071101 (2010).

[28] K. Hara et al. (Belle Collaboration), Phys. Rev. Lett. 110, 131801 (2013).

[29] J. P. Lees et al. (BaBar Collaboration), Phys. Rev. D88, 031102 (2013);

B. Aubert et al. (BaBar Collaboration), Phys. Rev. D81, 051101(R) (2010).

[30] A. Matyja et al. (Belle Collaboration), Phys. Rev. Lett. 99, 191807 (2007).

[31] A. Bozek et al. (Belle Collaboration), Phys. Rev. D82, 072005 (2010).

[32] I. Adachi et al. (Belle Collaboration), arXiv:0910.4301.

[33] J. P. Lees et al. (BaBar Collaboration), Phys. Rev. Lett. 109, 101802 (2012);

B. Aubert et al. (BaBar Collaboration), Phys. Rev. Lett. 100, 021801 (2008).

[34] T. Abe et al., arXiv:1011.0352.

[35] S. Hashimoto, M. Hazumi, J. Haba, J. Flanagan, and Y. Ohnishi, editors, Letter of Intent for KEK Super B Factory, 2004-04.

[36] I. Adachi et al., arXiv:0810.4084.

[37] T. Aushev et al., arXiv:1002.5012. 